\title{
Neurodegenerative diseases in a dish: the promise of iPSC technology in disease modeling and therapeutic discovery
}

\author{
Y. Z. Xie $\cdot$ R. X. Zhang
}

Received: 11 March 2014 / Accepted: 22 October 2014/Published online: 30 October 2014

(C) The Author(s) 2014. This article is published with open access at Springerlink.com

\begin{abstract}
The study of stem-cell biology has been a flourishing research area because of its multi-differentiation potential. The emergence of induced pluripotent stem cells (iPSCs) open up the possibility of addressing obstructs, such as the limited cell source, inherent complexity of the human brain, and ethical constrains. Though still at its infancy phase, reprogramming of somatic cells has been demonstrating the ability to enhance in vitro study of neurodegenerative diseases and potential treatment. However, iPSCs would not thoroughly translate to the clinic before limitations are addressed. In this review, by summarizing the recent development of iPSC-based models, we will discuss the feasibility of iPSC technology on relevant diseases depth and illustrate how this new tool applies to drug screening and celluar therapy.
\end{abstract}

Keywords Induced pluripotent stem cells · IPSCs · Neurodegenerative disease $\cdot$ Disease modeling $\cdot$ Drug screening $\cdot$ Cell therapy

\section{Introduction}

Neurodegenerative diseases occur as a result of neurodegenerative processes, including chronic and progressive loss of structure or function of neurons. Compared with other organs, recent progress in the molecular basis of neurodegenerative disorders has evolved slowly. This

\footnotetext{
Y. Z. Xie - R. X. Zhang $(\bowtie)$

Department of Neurology, The Third Xiangya Hospital, Central South University, Changsha 410013, Hunan Province, China e-mail: zhangruxu@vip.163.com

Y. Z. Xie

e-mail: yzxiexy3@163.com
}

mainly due to brain tissue samples were rarely available for obtaining and often demonstrated the final phase of the diseases. These limitations impede our progress for better understanding of disease onset and mechanisms [1]. Although genetically engineered animal models have achieved promising advances, these systems are still inadequate and do not faithfully mirror the disease mechanism due to species differences and genetic backgrounds. Possessing the developmental potential to form trophoblast and differentiate into three embryonic germ layers, human embryonic stem cells (hESCs) could be a new paradigm [2]. However, the success rate of hESC establishment is extremely low, combining with lacking of oocyte donors and ethical issues further restricted the extensive application. The recent method of obtaining induced pluripotent stem cells (iPSCs) from somatic cells offer unprecedented and exciting opportunities to solve multiple drawbacks of various models mentioned above. The aim of this review is to assess the recent literatures and key findings on modeling neurodegenerative disease using iPSCs. The potential of iPSCs as an ideal platform for drug screening and cell therapy are presented. Besides, limitations and challenges in iPSC modeling will also be discussed.

\section{The developmental process of iPSC technology}

Yamanaka first illustrated how cell fates rewound to a preembryonic state using retroviruses and lentiviruses by the ectopic co-expression of transcription factors [3, 4]. That discovery won him the Nobel Prize for Medicine in 2012. These iPS cells subsequently converted to specific cells of interest, such as neurons and glia that are relevant for different neurodegenerative diseases. However, it is also time-consuming, expensive. Moreover, this new approach 
possesses the possibility of oncogene reactivation when the cocktail of reprogramming factors contained proto oncogene $c-M Y C$ and the risk of insertional mutations. To reduce the potential adverse effects and to emphasize the need to safety, hiPSCs have been established by excluding $c-M Y C$ of four conventional reprogramming factors [5, 6]. Several groups took advantages of adenoviral and plasmid for non-integrating which avoid tumor formation, however, these manners are very labor intensive and the efficiency in general is extremely low [7, 8]. Sendai virus, protein, modified mRNA, and Micro-RNA have been used to generate iPSCs with no genomic integration [9-13]. The disadvantages of each vector type are still apparent. For example, the problems of purging cells of replicating virus and sequence-sensitive RNA replicase when applying Sendai virus can not be ignored [13]. Multiple rounds of transfection using modified mRNA vector were needed to achieve controllable and high-efficiency goal [12]. Recently, researchers have obtained mouse iPSCs from somatic cells using a combination of small-molecule compounds because they are cost-effective, easily synthesized, and nonimmunogenic [14]. Important progresses of iPSC-based scientific exploration have already been made on neurological diseases, haematological diseases, cardiac diseases, liver related diseases, etc. Here, we focus on current-established iPSCs models on neurodegenerative disorders (summarized in Table 1).

\section{Parkinson's disease}

Parkinson's disease (PD) is the most common form of a group of progressive neurodegenerative movement disorders, and the prevalence is about $100-300$ per 100,000 [15]. Initial PD-iPS cells were generated to reveal aspects of the abnormalities of PD, but no relevant phenotype was evident in the study [16]. Later, iPSC lines from a patient harboring a mutation in LRRK2 gene were found partially consistent with early PD phenotype of enhanced susceptibility to oxidative stress (OS) [17]. In LRRK2 G2019S iPSC-derived dopaminergic (DA) neurons, morphological

Table 1 Summary of reported iPSC-based disease models for neurodegenerative diseases

\begin{tabular}{|c|c|c|c|c|c|c|}
\hline Disease & $\begin{array}{l}\text { Associated } \\
\text { gene }\end{array}$ & Primary cells & Target cell differentiation & $\begin{array}{l}\text { Disease- } \\
\text { specific } \\
\text { phenotype }\end{array}$ & Therapeutic response & References \\
\hline \multirow[t]{5}{*}{ PD } & $L R R K 2$ & $\begin{array}{l}\text { Fibroblasts and } \\
\text { epidermal } \\
\text { keratinocytes }\end{array}$ & Dopaminergic neurons & Yes & Mutant allele correction & [17-19] \\
\hline & PINK1 & Fibroblasts & Dopaminergic neurons & Yes & PINK1 overexpression & {$[20]$} \\
\hline & PARK2 & Fibroblasts & Dopaminergic neurons & Yes & Lentiviral expression of Parkin & {$[21,22]$} \\
\hline & $S N C A$ & Fibroblasts & Dopaminergic neurons & Yes & Mutant allele correction & {$[23,24]$} \\
\hline & $G B A 1$ & Fibroblasts & Dopaminergic neurons & Yes & Mutant allele correction & {$[25]$} \\
\hline \multirow[t]{3}{*}{$\mathrm{AD}$} & $\begin{array}{l}P S 1 \text { and } \\
P S 2\end{array}$ & Fibroblasts & Neurons & Yes & $\begin{array}{l}\gamma \text {-Secretase inhibitors and } \\
\text { modulators }\end{array}$ & {$[28,29]$} \\
\hline & $A P P$ & Fibroblasts & Neurons & Yes & $\beta$-Secretase inhibitors & {$[30]$} \\
\hline & Sporadic & Fibroblasts & Neurons and astrocytes & Yes & Docosahexaenoic acid & {$[31]$} \\
\hline HD & $H T T$ & Fibroblasts & $\begin{array}{l}\text { Neural stem cells, neuronal } \\
\text { precursors striatal neurons, and } \\
\text { astrocytes }\end{array}$ & Yes & $\begin{array}{l}\text { Proteasome inhibitors and } \\
\text { normal repeat substitution }\end{array}$ & [34-39] \\
\hline \multirow[t]{3}{*}{ ALS } & SOD1 & Fibroblasts & Motor neurons & Yes & $\begin{array}{l}\text { Conditional expression of } \\
\text { neurofilament-L }\end{array}$ & {$[43,44]$} \\
\hline & $T D P-43$ & Fibroblasts & Motor neurons and astrocytes & Yes & Anacardic acid & $\begin{array}{l}{[45,46,} \\
48]\end{array}$ \\
\hline & $V A P B$ & Fibroblasts & Motor neurons & Yes & No & {$[47]$} \\
\hline SMA & SMN1 & $\begin{array}{l}\text { GM09677 } \\
\text { fibroblasts }\end{array}$ & Motor neurons & Yes & $\begin{array}{l}\text { Valproic acid, tobramycin, SMN } \\
\text { overexpressing and gene } \\
\text { correction }\end{array}$ & [51-55] \\
\hline FRDA & $F X N$ & Fibroblasts & $\begin{array}{l}\text { Peripheral sensory neurons and } \\
\text { neural crest progenitors }\end{array}$ & Yes & No & {$[60,61]$} \\
\hline MJD & $A T X N 3$ & Fibroblasts & Neurons & Yes & Calpain inhibition & {$[63]$} \\
\hline FD & $I K B K A P$ & Fibroblasts & $\begin{array}{l}\text { Neural crest precursors and } \\
\text { neurons }\end{array}$ & Yes & $\begin{array}{l}\text { Kinetin, glucosaminic acid, } \\
\text { SKF-86466, phenindione, etc. }\end{array}$ & {$[65-67]$} \\
\hline
\end{tabular}


alterations were observed when compared to control lines, with neurons showing decreased neurite length and reduced numbers of neuritis. This study also observed accumulation of autophagosomes and reduction of autophagic flux [18]. Recently, the phenotypes in two patient-derived iPSC lines with LRRK2 G2019S mutation were rescued by specifically correcting the corresponding mutant allele. Moreover, certain experiment highlighted an elevated level of $\alpha$ synuclein and MAPT expression in counterpart lines while not in the LRRK2 genetically corrected lines [19]. Mutations in either PINK1 or PARK2 cause recessive forms of inherited PD characterized by impaired mitophagy because PINK1 and Parkin proteins function together. A study, including three patients with missense (c.509T $>\mathrm{G}$; p.V170G) in mitochondrial protein PINK1, reported that the PINK1 mutant phenotype could be reversed after overexpression of wild-type PINKI [20]. In studies examining iPSC cells from patients with PARK2 mutations, iPSC-derived neurons demonstrated functional and morphological abnormalities of mitochondria along with increased oxidative stress, rather than iPSCs or fibroblasts [21]. Similar to Imaizumi and colleagues, Jiang et al. [22] demonstrated altered features in mutant DA neurons, including enhanced sensibility to OS and monoamine oxidases activity, increased spontaneous DA release, and decreased DA uptake. These phenotypes were reversed through lentiviral expression of Parkin. Human iPSCderived lines with mutations in SNCA expressed double the amount of $\alpha$-synuclein when differentiated into DA neurons, while not seen in the original fibroblasts [23]. By use of zinc finger nuclease-mediated genome editing technology, researchers corrected the underlying point mutations (A53T) in SNCA-PD-iPSC. However, the phenotypes of iPSC-derived neurons in this work were not assessed [24]. A more recent study applied iPSCs possessing GBAl mutations to the modeling of PD. Schondorf et al. [25] observed increased levels of $\alpha$-synuclein and glucosylceramide as well as lysosomal and autophagic defects in neurons from GBAI PD-iPSC. Importantly, these pathological phenotypes could be rescued by correction of the mutations.

\section{Alzheimer's disease}

Alzheimer's disease (AD) is caused by progressive degeneration and loss of neurons and synapses throughout the brain [26]. Most of early-onset familial AD (FAD) can be attributed to mutations in one of three genes: APP, PS1 and $P S 2$, while many genetic and environmental factors may co-contribute to determining the sporadic AD (SAD) [27]. The investigation of $\mathrm{AD}$ was extremely limited until FAD-derived iPSCs with PS1 (A246E) and PS2 (N141I) mutations were established. In FAD-iPSC-derived differentiated neurons, the secretion of amyloid-beta $(A \beta)$ was significantly increased and sharply responds to $\gamma$ secretase modulators and inhibitors [28]. Furthermore, iPSC-derived neuronal cells showed AD-like biochemical features, such as an increase in $A \beta$ ratio. These neurons have reduced to secrete $A \beta$ when treated with $\beta$-secretase inhibitor, $\gamma$-secretase inhibitor, and a nonsteroidal antiinflammatory drug. During the differentiation stages, however, there existed different susceptibilities to these drugs [29]. Other iPSCs model was established from FAD patients with a duplication of $A P P$ gene and two cases of SAD. Higher level of phospho-tau and glycogen synthase kinase- 3 in lines with elevated $A \beta$ represented novel observations in neurons from one of the SAD cases. Interestingly, these alterations could be alleviated by $\beta$ secretase, not $\gamma$-secretase, inhibitor treatment [30]. Docosahexaenoic acid (DHA) may actually be effective for some patients for the reason that the stress responses in the AD neural cells were alleviated when treated with DHA, making possible the iPSC technology for validation and identification of promising drugs [31].

\section{Huntington's disease}

Huntington's disease (HD) is a progressive neurodegenerative genetic disease associated with a wide variety of motor impairment and psychiatric symptoms caused by excessive CAG trinucleotide expansions of Huntingtin gene (HTT) [32]. The first established human HD-iPSC model from HD patients carrying 72 CAG repeats was reported in 2008 [33]. Striatal neurons and HD neural stem cells produced from iPSCs exhibited elevated caspase activity after the withdrawal of growth factors which indicate apoptosis, but no overt cell death phenotype was reported [34]. Experimental analysis of proteins revealed amounts of distinct alternations in protein expression in the HD iPSCs [35]. Jeon et al. converted HD-iPSC into GABAergic striatal neurons and relavent behavior recovered in a grafted rat after transplantation of HD-iPSC derived neural precursors. Though showing no overt HD phenotype, the disease-specific iPSCs sharply responded to proteasome inhibition and expressed several markers of HD pathology after transplantation at later time points [36, 37]. Additional HD-phenotypes, including altered mitochondrial bioenergetics and susceptibility to cell death were reported in a subsequent study. These alterations accompanied with pathogenic HD signaling pathways could be reversed by the substitution of a normal repeat for the expanded CAG repeat using homologous recombination [38]. A more complete study by the HD Consortium demonstrated hundreds of distinct differences between HD and wide-type (WT) iPSCs, showing clear signs of HDrelated pathology in several lineages. The lines with the 
longer CAG repeat expansions had more severe pathological phenotypes and ultimately lead to neuronal death [39]. These findings revealed that HD-iPSCs could be a wellcharacterized and unique resource to elucidate disease mechanisms.

\section{Amyotrophic lateral sclerosis}

Amyotrophic lateral sclerosis (ALS) is the most common form of progressive motor neuron disorder with varied etiology characterized by rapidly progressive weakness and muscle atrophy. This disease strikes people in their 40s, approximately $20 \%$ of familial ALS cases were linked to dominant mutations in the SODI gene [40]. Other different genes, including $V A P B, T D P-43$ and $F U S$ have also been implicated in ALS [41, 42]. ALS-iPSCs were developed using skin fibroblasts from two octogenarian sisters with SOD1 mutations and then converted into spinal motor neurons, but no assay of ALS relevant phenotype or compared with neurons from controls were performed in this study [43]. Another iPSC-derived motor neurons (MNs) possessing SOD1 mutations developed neurofilament (NF) inclusions. Importantly, conditional expression of NF-L in these MNs corrected the NF subunit proportion, mitigating NF aggregation and neurite degeneration [44]. The mutant astrocytes from iPSCs harboring a mutation in the TDP-43 gene exhibited subcellular mislocalization of TDP-43, increased levels of TDP-43, and reduced cell survival. Further co-culture assays showed these astrocytes did not adversely affect survival of cocultured neurons [45]. Another TDP-43 iPSC-derived MNs recapitulated the disease phenotypes, including decreased survival, increased cellular vulnerability, and elevated levels of soluble and detergent-resistant TDP-43 protein [46]. Researchers also generated iPSCs from patients which carry the $V A P B$ mutation as well as from their healthy siblings. The finding suggested that reduced levels of VAPB protein in MNs could be involved in the pathogenesis of ALS8 [47]. Test for chemical compounds on differentiated MNs showed that the abnormal ALS MNs phenotype could be rescued by anacardic acid, suggesting that special MNs may be a new approach for elucidating ALS disease mechanisms and for screening candidate drugs [48].

\section{Spinal muscular atrophy}

Spinal muscular atrophy (SMA) is an autosomal recessive genetic disease characterized by the loss of the survival motor neuron (SMN) [49]. There are two SMN genes in humans, SMN1 and SMN2, and the loss of the SMN1 gene primarily caused SMA [50]. To model SMA, scientists generated iPSCs from a child carrying SMN1 mutation.
These cells manifested clear signs of motor neurons and differentiated neurons showed the reduction in cells size and number compared to controls at later stages, indicating they underwent substantial degeneration. Excitingly, the administration of valproic acid and tobramycin could reverse the endogenous SMN protein level in differentiated neurons and astrocytes [51]. Another established neuronal cultures rescued the phenotype of delayed neurite outgrowth and restored normal motoneuron differentiation by overexpressing SMN, accelerating the exploration of the underlying mechanisms of SMA pathogenesis [52]. MNs from two type I SMA subject-derived iPSCs showed elevated activation of caspase- 8 and- 3 and increased Fas ligand-mediated apoptosis. Moreover, distinct inhibitors of apoptotic pathways may reduce motor neuron cell death [53]. Another study has focused on genetic correction for autologous cell therapy. Uncorrected SMA-iPSCs derived motor neurons manifested disease-specific features. Delightfully, these phenotypes were ameliorated in genetically corrected controls, suggesting that genetically corrected special motor neurons could be a source for future therapeutic strategy [54]. Novel observations of reduced androgen receptor levels, reduced HDAC6, and repeat instability provided evidence that SMA-iPSC derived MNs could be new avenues for further investigation of the disease mechanism and development of effective therapy [55].

\section{Inherited ataxias}

Inherited ataxias may show autosomal dominant, autosomal recessive modes of inheritance. Friedreich ataxia (FRDA) is an autosomal recessive ataxia, accounting for one-half of all hereditary ataxias. Spinocerebellar ataxias (SCAs) are genetically defined autosomal dominantly inherited disorders characterized by progressive lack of motor coordination.

FRDA is caused by large guanine-adenine-adenine (GAA) expansions in $F X N$ gene on chromosome 9q13, leading to a transcriptional defect of FXN mRNA and frataxin $[56,57]$. GAA-TTC triplet repeats in $F X N$ intron 1 in FRDA iPSCs not only expanded at a higher rates but also exhibited repeat instability [58, 59]. The mismatch repair enzymes $\mathrm{MSH} 2$ were much highly expressed in iPSCs than fibroblasts and neuronal stem cells. Moreover, a specific pyrrole-imidazole polyamide which displaced MSH2 in FRDA iPSCs could partially impede repeat expansion [59]. Studies above demonstrated that GAA repeats instability might start early during ontogenesis and the highly active mismatch repair system is related to the GAA.TTC triplet repeats. Another iPSCs presented an instable repeats of GAA expansion, but was failed to find biochemical phenotypes [60]. Recently, a new development-based differentiation protocol was applied to 
differentiate FRDA and control iPSCs into peripheral sensory neurons and neural crest progenitors. Increased expression of frataxin during sensory specification for control cells was identified compared to FRDA peripheral sensory neurons. Whereas, a pronounced deficiency of frataxin was observed in FRDA iPSCs and neural crest cells, rather than controls [61].

SCA3, also known as Machado-Joseph disease, is the most common subtype of SCAs in China, which is caused by an abnormal CAG trinucleotide repeat expansion in ATXN3 gene [62]. The formation of sodium dodecyl sulfate insoluble aggregates was observed in L-glutamate-induced excitation of iPSC-derived neurons and calpain inhibition could abolish these pathological changes. However, inclusion bodies and increased cell death were not observed, suggesting the excitation-induced protein aggregation is an early event [63].

\section{Familial dysautonomia}

Familial dysautonomia (FD) is a debilitating neurodegenerative disease and the most common mutation is in a splice site of the IKBKAP gene involved in transcriptional elongation, resulting in reduced levels of IKAP protein [64]. Researchers differentiated patient-specific FD-iPSCs into peripheral neurons directly. Tissue-specific mis-splicing of IKBKAP in vitro and low level of normal IKBKAP transcript expressed in patient neural crest precursors were observed. The potency treatment of rescuing the mutant splice combines with improving neuronal differentiation and migration were also concluded $[65,66]$. Later, 6,912 compounds were screened, while 8 hits were characterized that rescued expression of IKBKAP. SKF-86466, one of 8 hits, not only induced IKBKAP transcription but also rescued the expression of IKAP protein and the disease-specific loss of autonomic neuron sign [67]. Taken together, small molecule discovery in these newly disease models can gain novel insights into human disease pathogenesis and promising treatment.

\section{Potential and challenges}

The promising iPSC technology has the potential to model and treat neurodegenerative diseases. Patient-derived iPSCs can be rewound to affected neuronal subtypes via in vitro differentiation or repaired iPS cells using gene targeting to repair disease-causing mutation. One notable potential is to apply these affected neuronal subtypes to develop promising drugs that are most suitable for the patient associated with their efficacy and toxicity profile. The generation of iPSCs permits the production of large scale of central nervous system cell with specific genotype, providing an unlimited amount of experimental materials. The other potential is to develop autologous-repaired iPS cells for cell therapy. When transplanting desired cells into patients, neural function could be restored and long-term immuno-suppression may not be necessary. Moreover, transplantation of glial cells, such as astrocytes that would restore the motor neurons potentially, can also used for neuroprotection.

However, it is not until this technology ready for clinical applications that its limitations were overcame. Firstly, initial method that introduction of factors by retrovirus or lentivirus might provide the possibility of oncogene reactivation [3, 4]. Apart from safety, the genetic and epigenetic status of clones might have varied after reprogramming. Although the non-integrating approaches significantly reduced the risk of tumorigenesis and epigenetic variation, researchers need to be cautious and select the stable lineages for differentiation studies. Epigenetic modifications could be significant in disease manifestation and the erasure of epigenetic marks during reprogramming is important [713]. Secondly, how to generate disease-relevant phenotype in vitro is also a challenge. The appearance of disease phenotype is the most important feature for disease modeling. Current iPSC-models were generated from patients containing mutations in known gene. However, many patients, such as PD and ALS, have an unidentified genetic component that is coupled with environmental factors. Importantly, the majority of neurodegenerative disorders are age-dependent and age is considered to be a risk factor that contributes to the disease development. As current culture system involved no disease-triggering factors on disease, the iPSCs might no longer reflect relevant pathogenesis. Moreover, long-term culture was poorly controlled and could be compromised by excessive cell death. Thirdly, protocols for differentiating iPSCs towards different subtypes of neurons cannot be ignored. Up till now, greater progress has been made in generating matured populations of distinct neurons and glia for the purpose of screening drugs and replacement therapy. However, these cells may not truly reflect the cellular responses to compounds that the body would have at a physiological level. Lastly, researchers still face the problems of low efficiency conversion and laborious to conduct. The average conversion efficiency of each methods mentioned above is less than $1 \%$, which further restrict the extensively use of the technology [1]. Efficiency needs to be improved if these protocols apply for high-throughout drug screening.

\section{Conclusions}

Taken together, iPSC technology for modeling of neurodegenerative diseases has both benefits and limitations. Patient-derived cells open new avenues to investigate the 
neurodegenerative diseases development and remarkably easily could usher in new therapies and cloning techniques. Many hurdles must be overcome before new clinical therapies based on these cells. This will help ensure safety and eventually beneficial to human beings.

Acknowledgments This work was supported by research grants from the Hunan Provincial Natural Science Foundation of China (13JJ2014) and National Natural Science Foundation of China (81071001).

Open Access This article is distributed under the terms of the Creative Commons Attribution License which permits any use, distribution, and reproduction in any medium, provided the original author(s) and the source are credited.

\section{References}

1. Robinton DA, Daley GQ (2012) The promise of induced pluripotent stem cells in research and therapy. Nature 481:295-305

2. Thomson JA, Itskovitz-Eldor J, Shapiro SS, Waknitz MA, Swiergiel JJ, Marshall VS, Jones JM (1998) Embryonic stem cell lines derived from human blastocysts. Science 282:1145-1147

3. Takahashi K, Tanabe K, Ohnuki M, Narita M, Ichisaka T, Tomoda K, Yamanaka S (2007) Induction of pluripotent stem cells from adult human fibroblasts by defined factors. Cell 131:861-872

4. Takahashi K, Yamanaka S (2006) Induction of pluripotent stem cells from mouse embryonic and adult fibroblast cultures by defined factors. Cell 126:663-676

5. Huangfu D, Osafune K, Maehr R et al (2008) Induction of pluripotent stem cells from primary human fibroblasts with only Oct4 and Sox2. Nat Biotechnol 26:1269-1275

6. Nakagawa M, Koyanagi M, Tanabe K et al (2008) Generation of induced pluripotent stem cells without Myc from mouse and human fibroblasts. Nat Biotechnol 26:101-106

7. Okita K, Nakagawa M, Hyenjong H, Ichisaka T, Yamanaka S (2008) Generation of mouse induced pluripotent stem cells without viral vectors. Science 322:949-953

8. Stadtfeld M, Nagaya M, Utikal J, Weir G, Hochedlinger K (2008) Induced pluripotent stem cells generated without viral integration. Science 322:945-949

9. Zhou H, Wu S, Joo JY et al (2009) Generation of induced pluripotent stem cells using recombinant proteins. Cell Stem Cell 4:381-384

10. Heng BC, Heinimann K, Miny P et al (2013) mRNA transfectionbased, feeder-free, induced pluripotent stem cells derived from adipose tissue of a 50-year-old patient. Metab Eng 18:9-24

11. Wang T, Shi SB, Sha HY (2013) Micro RNAs in regulation of pluripotency and somatic cell reprogramming: small molecule with big impact. RNA Biol 10:1255-1261

12. Warren L, Manos PD, Ahfeldt T et al (2010) Highly efficient reprogramming to pluripotency and directed differentiation of human cells with synthetic modified mRNA. Cell Stem Cell 7:618-630

13. Fusaki N, Ban H, Nishiyama A, Saeki K, Hasegawa M (2009) Efficient induction of transgene-free human pluripotent stem cells using a vector based on Sendai virus, an RNA virus that does not integrate into the host genome. Proc Jpn Acad Ser B Phys Biol Sci 85:348-362

14. Hou P, Li Y, Zhang X et al (2013) Pluripotent stem cells induced from mouse somatic cells by small-molecule compounds. Science 341:651-654
15. Lees AJ, Hardy J, Revesz T (2009) Parkinson's disease. Lancet 373:2055-2066

16. Soldner F, Hockemeyer D, Beard C et al (2009) Parkinson's disease patient-derived induced pluripotent stem cells free of viral reprogramming factors. Cell 136:964-977

17. Nguyen HN, Byers B, Cord B et al (2011) LRRK2 mutant iPSCderived DA neurons demonstrate increased susceptibility to oxidative stress. Cell Stem Cell 8:267-280

18. Sanchez-Danes A, Richaud-Patin Y, Carballo-Carbajal I et al (2012) Disease-specific phenotypes in dopamine neurons from human iPS-based models of genetic and sporadic Parkinson's disease. EMBO Mol Med 4:380-395

19. Reinhardt P, Schmid B, Burbulla LF et al (2013) Genetic correction of a LRRK2 mutation in human iPSCs links parkinsonian neurodegeneration to ERK-dependent changes in gene expression. Cell Stem Cell 12:354-367

20. Seibler P, Graziotto J, Jeong H, Simunovic F, Klein C, Krainc D (2011) Mitochondrial Parkin recruitment is impaired in neurons derived from mutant PINK1 induced pluripotent stem cells. J Neurosci 31:5970-5976

21. Imaizumi Y, Okada Y, Akamatsu W et al (2012) Mitochondrial dysfunction associated with increased oxidative stress and alphasynuclein accumulation in PARK2 iPSC-derived neurons and postmortem brain tissue. Mol Brain 5:35

22. Jiang H, Ren Y, Yuen EY et al (2012) Parkin controls dopamine utilization in human midbrain dopaminergic neurons derived from induced pluripotent stem cells. Nat Commun 3:668

23. Devine MJ, Ryten M, Vodicka P et al (2011) Parkinson's disease induced pluripotent stem cells with triplication of the alphasynuclein locus. Nat Commun 2:440

24. Soldner F, Laganiere J, Cheng AW et al (2011) Generation of isogenic pluripotent stem cells differing exclusively at two early onset Parkinson point mutations. Cell 146:318-331

25. Schondorf DC, Aureli M, McAllister FE et al (2014) iPSCderived neurons from GBA1-associated Parkinson's disease patients show autophagic defects and impaired calcium homeostasis. Nat Commun 5:4028

26. Ballard C, Gauthier S, Corbett A, Brayne C, Aarsland D, Jones E (2011) Alzheimer's disease. Lancet 377:1019-1031

27. Piaceri I, Nacmias B, Sorbi S (2013) Genetics of familial and sporadic Alzheimer's disease. Front Biosci (Elite Ed) 5:167-177

28. Yagi T, Ito D, Okada Y et al (2011) Modeling familial Alzheimer's disease with induced pluripotent stem cells. Hum Mol Genet 20:4530-4539

29. Yahata N, Asai M, Kitaoka S et al (2011) Anti-Abeta drug screening platform using human iPS cell-derived neurons for the treatment of Alzheimer's disease. PLoS One 6:e25788

30. Israel MA, Yuan SH, Bardy C et al (2012) Probing sporadic and familial Alzheimer's disease using induced pluripotent stem cells. Nature 482:216-220

31. Kondo T, Asai M, Tsukita K et al (2013) Modeling Alzheimer's disease with iPSCs reveals stress phenotypes associated with intracellular Abeta and differential drug responsiveness. Cell Stem Cell 12:487-496

32. Ha AD, Fung VS (2012) Huntington's disease. Curr Opin Neurol 25:491-498

33. Park IH, Arora N, Huo H et al (2008) Disease-specific induced pluripotent stem cells. Cell 134:877-886

34. Zhang N, An MC, Montoro D, Ellerby LM (2010) Characterization of human Huntington's disease cell model from induced pluripotent stem cells. PLoS Curr 2:Rrn1193

35. Chae JI, Kim DW, Lee N et al (2012) Quantitative proteomic analysis of induced pluripotent stem cells derived from a human Huntington's disease patient. Biochem J 446:359-371 
36. Jeon I, Lee N, Li JY et al (2012) Neuronal properties, in vivo effects, and pathology of a Huntington's disease patient-derived induced pluripotent stem cells. Stem Cells 30:2054-2062

37. Jeon I, Choi C, Lee $\mathrm{N}$ et al (2014) In vivo roles of a patientderived induced pluripotent stem cell line (HD72-iPSC) in the YAC128 model of Huntington's disease. Int J Stem Cells 7:43-47

38. An MC, Zhang N, Scott G et al (2012) Genetic correction of Huntington's disease phenotypes in induced pluripotent stem cells. Cell Stem Cell 11:253-263

39. HD iPSC Consortium (2012) Induced pluripotent stem cells from patients with Huntington's disease show CAG-repeat-expansionassociated phenotypes. Cell Stem Cell 11:264-278

40. Turner MR, Hardiman O, Benatar M et al (2013) Controversies and priorities in amyotrophic lateral sclerosis. Lancet Neurol $12: 310-322$

41. Aliaga L, Lai C, Yu J et al (2013) Amyotrophic lateral sclerosisrelated VAPB P56S mutation differentially affects the function and survival of corticospinal and spinal motor neurons. Hum Mol Genet 22(21):4293-4305

42. Al-Chalabi A, Jones A, Troakes C, King A, Al-Sarraj S, van den Berg LH (2012) The genetics and neuropathology of amyotrophic lateral sclerosis. Acta Neuropathol 124:339-352

43. Dimos JT, Rodolfa KT, Niakan KK et al (2008) Induced pluripotent stem cells generated from patients with ALS can be differentiated into motor neurons. Science 321:1218-1221

44. Chen H, Qian K, Du Z et al (2014) Modeling ALS with iPSCs reveals that mutant SOD1 misregulates neurofilament balance in motor neurons. Cell Stem Cell 14:796-809

45. Serio A, Bilican B, Barmada SJ et al (2013) Astrocyte pathology and the absence of non-cell autonomy in an induced pluripotent stem cell model of TDP-43 proteinopathy. Proc Natl Acad Sci USA 110:4697-4702

46. Bilican B, Serio A, Barmada SJ et al (2012) Mutant induced pluripotent stem cell lines recapitulate aspects of TDP-43 proteinopathies and reveal cell-specific vulnerability. Proc Natl Acad Sci USA 109:5803-5808

47. Mitne-Neto M, Machado-Costa M, Marchetto MC et al (2011) Downregulation of VAPB expression in motor neurons derived from induced pluripotent stem cells of ALS8 patients. Hum Mol Genet 20:3642-3652

48. Egawa N, Kitaoka S, Tsukita K et al (2012) Drug screening for ALS using patient-specific induced pluripotent stem cells. Sci Trans Med 4:145ra104

49. Hamilton G, Gillingwater TH (2013) Spinal muscular atrophy: going beyond the motor neuron. Trends Mol Med 19:40-50

50. Nurputra DK, Lai PS, Harahap NI et al (2013) Spinal muscular atrophy: from gene discovery to clinical trials. Ann Hum Genet 77(5):435-463

51. Ebert AD, Yu J, Rose FF Jr, Mattis VB, Lorson CL, Thomson JA, Svendsen CN (2009) Induced pluripotent stem cells from a spinal muscular atrophy patient. Nature 457:277-280

52. Chang T, Zheng W, Tsark W, Bates S, Huang H, Lin RJ, Yee JK (2011) Brief report: phenotypic rescue of induced pluripotent stem cell-derived motoneurons of a spinal muscular atrophy patient. Stem Cells 29:2090-2093

53. Sareen D, Ebert AD, Heins BM, McGivern JV, Ornelas L, Svendsen CN (2012) Inhibition of apoptosis blocks human motor neuron cell death in a stem cell model of spinal muscular atrophy. PLoS One 7:e39113

54. Corti S, Nizzardo M, Simone C et al (2012) Genetic correction of human induced pluripotent stem cells from patients with spinal muscular atrophy. Sci Trans Med 4:165ra162

55. Grunseich C, Zukosky K, Kats IR et al (2014) Stem cell-derived motor neurons from spinal and bulbar muscular atrophy patients. Neurobiol Dis 70:12-20

56. Koeppen AH (2011) Friedreich's ataxia: pathology, pathogenesis, and molecular genetics. J Neurol Sci 303:1-12

57. Lufino MM, Silva AM, Nemeth AH, Alegre-Abarrategui J, Russell AJ, Wade-Martins R (2013) A GAA repeat expansion reporter model of Friedreich's ataxia recapitulates the genomic context and allows rapid screening of therapeutic compounds. Hum Mol Genet 22(25):5173-5187

58. Ku S, Soragni E, Campau E et al (2010) Friedreich's ataxia induced pluripotent stem cells model intergenerational GAATTC triplet repeat instability. Cell Stem Cell 7:631-637

59. Du J, Campau E, Soragni E, Ku S, Puckett JW, Dervan PB, Gottesfeld JM (2012) Role of mismatch repair enzymes in GAATTC triplet-repeat expansion in Friedreich ataxia induced pluripotent stem cells. J Biol Chem 287:29861-29872

60. Hick A, Wattenhofer-Donze M, Chintawar S et al (2013) Neurons and cardiomyocytes derived from induced pluripotent stem cells as a model for mitochondrial defects in Friedreich's ataxia. Dis Model Mech 6:608-621

61. Eigentler A, Boesch S, Schneider R, Dechant G, Nat R (2013) Induced pluripotent stem cells from friedreich Ataxia patients fail to up-regulate frataxin during in vitro differentiation to peripheral sensory neurons. Stem Cells Dev 22(24):3271-3282

62. Costa Mdo C, Paulson HL (2012) Toward understanding Machado-Joseph disease. Prog Neurobiol 97:239-257

63. Koch P, Breuer P, Peitz M et al (2011) Excitation-induced ataxin3 aggregation in neurons from patients with Machado-Joseph disease. Nature 480:543-546

64. Slaugenhaupt SA, Blumenfeld A, Gill SP et al (2001) Tissuespecific expression of a splicing mutation in the IKBKAP gene causes familial dysautonomia. Am J Hum Genet 68:598-605

65. Lee G, Papapetrou EP, Kim H et al (2009) Modelling pathogenesis and treatment of familial dysautonomia using patientspecific iPSCs. Nature 461:402-406

66. Lee G, Studer L (2011) Modelling familial dysautonomia in human induced pluripotent stem cells. Philos Trans R Soc Lond B Biol Sci 366:2286-2296

67. Lee G, Ramirez CN, Kim H et al (2012) Large-scale screening using familial dysautonomia induced pluripotent stem cells identifies compounds that rescue IKBKAP expression. Nat Biotechnol 30:1244-1248 